POS $\quad \begin{aligned} & \text { PROCEEDINGS } \\ & \text { OF SCIENCE }\end{aligned}$

\title{
Neutrino oscillations and PMNS unitarity with IceCube-DeepCore and the IceCube Upgrade
}

\section{Tom Stuttard for the IceCube collaboration*}

Niels Bohr Institute, Blegdamsvej 17, 2100 Copenhagen, Denmark.

E-mail: stuttardeicecube.wisc.edu

The $3 v$ paradigm can be tested with precision measurements of the unitarity of the Pontecorvo-Maki-Nakagawa-Sakata (PMNS) neutrino mixing matrix. However, such tests are hampered by the low precision of $v_{\tau}$ oscillation measurements, and new measurements are required. A recent world-leading measurement of $v_{\tau}$ appearance by the DeepCore sub-array of the IceCube neutrino observatory is presented, as well as the future prospects from the upcoming IceCube Upgrade.

The 21st international workshop on neutrinos from accelerators (NuFact2019) August 26 - August 31, 2019

Daegu, Korea

${ }^{*}$ Speaker. 


\section{PMNS unitarity and $v_{\tau}$}

The observation of neutrino oscillations, where a neutrino produced as one flavor may be detected some time later as another, implies mixing between the neutrino flavor and mass states, as well as non-zero masses for at least two of the neutrino mass states. For the three known neutrino flavor states (electron, $\mu$ and $\tau$ ), this mixing is characterised by the Pontecorvo-Maki-Nakagawa - Sakata (PMNS) $3 \times 3$ matrix [1,2]. The PMNS matrix is expected to be unitary, meaning that it completely describes the mixing between states, and any deviation from unitarity could indicate the presence of Beyond Standard Model (BSM) physics, including additional 'sterile' neutrinos states. Measurements of PMNS matrix unitarity therefore provide a powerful and model-independent test of the $3 v$ paradigm.

Testing the unitarity of the PMNS matrix requires measurements of all 9 matrix elements, ideally via measurements of all possible neutrino oscillation channels (e.g. $v_{e} \rightarrow v_{e}, v_{e} \rightarrow v_{\mu}$, etc). However, the vast majority of global neutrino oscillation measurements are derived from the appearance or disappearance of electron or $\mu$ neutrinos, whilst the $\tau$ sector remains relatively unexplored [3].

This lack of experimental data constraining the $\tau$ sector of the PMNS matrix significantly reduces the precision with which its unitarity can be tested, and is a consequence of the large $\tau$ lepton mass, which forbids $v_{\tau}$ production via pion or kaon decays and suppresses the charged current (CC) $v_{\tau}$ cross section below $\sim 1 \mathrm{TeV}$. Worse still, the short lifetime of the $\tau$ produced in CC interactions causes it to decay after travelling only microscopic distances in a detector, hampering identification of $v_{\tau}$ events.

To date, three experiments have been able to measure $v_{\tau}$ oscillations via the $v_{\mu} \rightarrow v_{\tau}$ channel ( $v_{\tau}$ appearance), albeit with precision significantly below that achieved in $v_{e}$ and $v_{\mu}$ measurements. The OPERA experiment observed $10 v_{\tau}$ candidates appearing in the CNGS $v_{\mu}$ beam [4], identified via the $\tau$ lepton decay topology utilising an emulsion cloud chamber detector to achieve sub-mm spatial precision. Separately, the Super-Kamiokande [5] and IceCube [6] collaborations have exploited the large naturally occurring flux of neutrinos produced by cosmic ray interactions with the Earth's atmosphere. Atmospheric $v_{\mu}$ with $\sim 25 \mathrm{GeV}$ crossing the Earth's diameter maximally oscillate to $v_{\tau}$, and at these energies the suppression to the $\mathrm{CC} v_{\tau}$ cross section is $\mathscr{O}(50 \%)$ relative to the $v_{e, \mu}$ cross sections [7], resulting in significant numbers of $v_{\tau}$ appearance events being observed in these massive underground detectors. The $v_{\tau}$ are not individually identified, but the appearance of many $v_{\tau}$ is observed statistically.

\section{2. $v_{\tau}$ appearance in IceCube-DeepCore}

The IceCube neutrino observatory [8] consists of 5160 Digital Optical Modules (DOMs) instrumenting a cubic-km of glacial ice deep below the geographic South Pole, with each DOM containing a single photomultiplier tube (PMT). Via the Cherenkov light produced by the charged secondaries of $v$-ice interactions, this vast 1 Gton instrument observes huge rates of neutrinos of atmospheric and astrophysical origin. The 10 Mton DeepCore [9] sub-array in the deepest, clearest ice is instrumented more densely, lowering the detection threshold to $\sim 5 \mathrm{GeV}$ and providing sensitivity to $v_{\tau}$ appearance. 
Neutrino oscillations are measured in DeepCore by reconstructing the energy and zenith angle (a proxy for neutrino travel distance) of the detected neutrinos, and comparing the flavor composition to the expected atmospheric neutrino flux. The flavor composition cannot be fully reconstructed, but crucially $v_{\mu}$ CC interactions can be identified due to the elongated track-like topology of the deposited light produced by the long-lived $\mu$ produced, in contrast to the more spherical cascade-like topology observed for all other neutrino interactions.

The $v_{\tau}$ appearance measurement is performed by fitting Monte Carlo (MC) simulation to detector data, scaling the $v_{\tau}$ contribution. This scaling is known as the $v_{\tau}$ normalisation, $N_{v_{\tau}}$, with a value of 1 expected for a unitary PMNS matrix. Nuisance parameters representing the uncertainty in neutrino oscillation parameters, atmospheric neutrino flux, neutrino interaction cross sections, optical sensor efficiency, ice properties and atmospheric muon background are included.

The results of two independent DeepCore $v_{\tau}$ appearance measurements [6] are shown Figure 1. Three years of DeepCore data were used, achieving world-leading precision. The lead analysis observed a total of $1804 \pm 9 \mathrm{CC}$ events at the best fit point, an order of magnitude more than any previous measurement, corresponding to a $v_{\tau}$ normalisation of $0.73_{-0.24}^{+0.30}$, consistent with the expectation for a unitary PMNS matrix within $1 \sigma$. The second analysis obtains a consistent result. The DeepCore results indicate a lower $v_{\tau}$ normalisation than measured by OPERA and SuperKamiokande, though the tension is mild given the large uncertainties. A new measurement using 8 years of DeepCore data is currently underway.

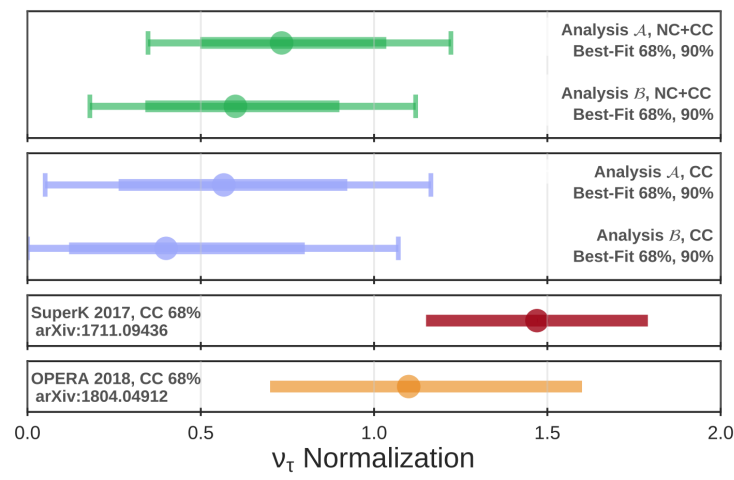

Figure 1: Measured $v_{\tau}$ normalisation, including $1 \sigma$ and $90 \%$ uncertainty, in two DeepCore analyses using 3 years of data [6]. The results of the Super-Kamiokande [5] and OPERA [4] experiments are shown for comparison.

The $v_{\tau}$ normalisation can also be interpreted as a deviation of the $v_{\tau} \mathrm{CC}$ cross section relative to the theoretical prediction. This cross section is poorly known experimentally, although shares many theoretical similarities with the much more precisely measured $v_{\mu} \mathrm{CC}$ cross section.

\section{The IceCube Upgrade}

The IceCube Upgrade, to be deployed in 2022-23, will feature $\sim 700$ new and enhanced multiPMT optical sensors in a dense configuration within the already dense DeepCore array, as well as a suite of new calibration devices, and will be utilised to perform precision neutrino oscillation measurements. The increased light observed from neutrino interactions in the new detector is 

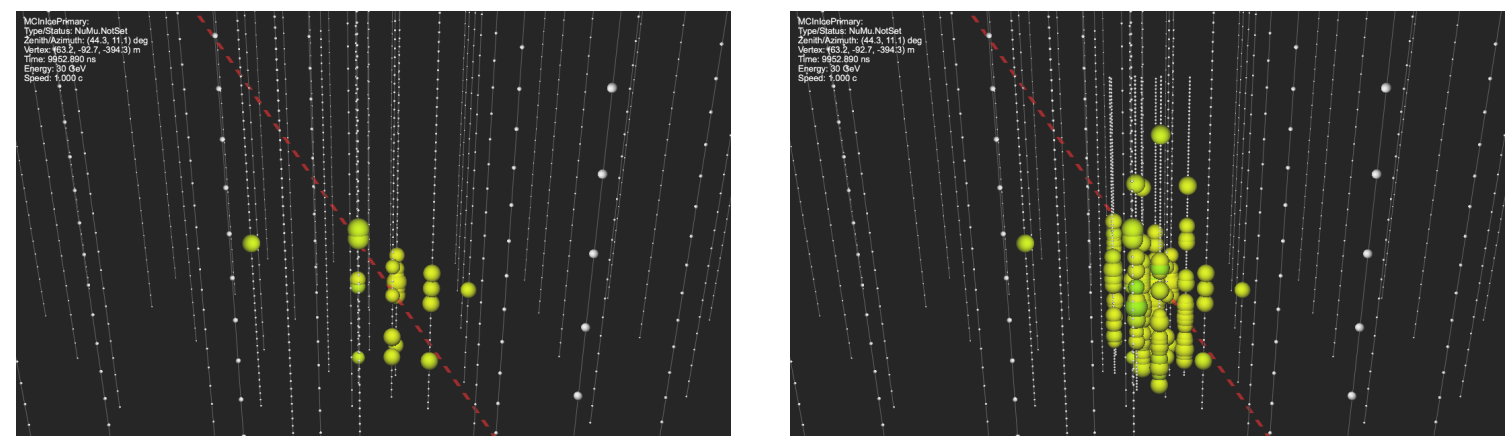

Figure 2: Event displays showing the same $30 \mathrm{GeV} v_{\mu} \mathrm{CC}$ interaction in the DeepCore (left) and IceCube Upgrade (right) sub-arrays. The white spheres represent optical sensors, whilst the colored spheres indicate photon hits on the sensors. The red line shows the path of the neutrino.

shown in Figure 2, along with the existing DeepCore array. This will significantly increase the rate of detected neutrinos in the DeepCore energy range, as well as lowering the detection threshold to provide high statistics measurements of $\sim 1 \mathrm{GeV}$ neutrinos, probing the second neutrino oscillation maximum and potentially providing sensitivity to the neutrino mass ordering via matter effects.

The performance of reconstructions of the neutrino energy and direction will be significantly enhanced in this new detector, with a factor 3 improvement in cascade zenith angle resolution at $\sim 25 \mathrm{GeV}$ expected, shown in Figure 3. The ability to identify $v_{\mu} \mathrm{CC}$ track-like events will also be improved.
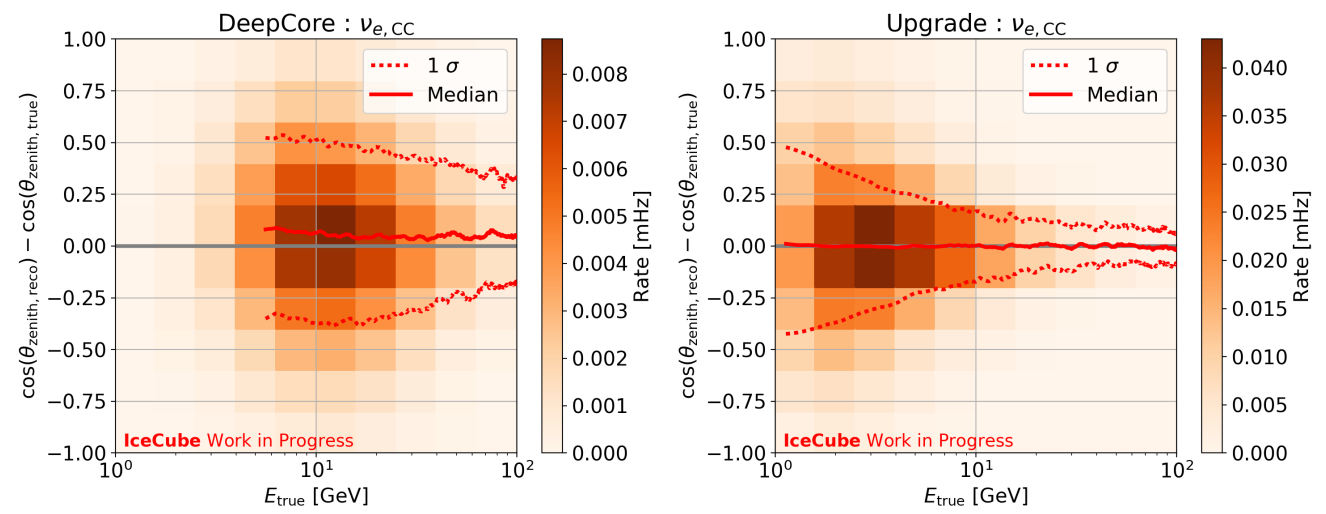

Figure 3: Histogram of the zenith angle reconstruction error for $v_{e} \mathrm{CC}$ (cascade-like) events in DeepCore (left) and the IceCube Upgrade (right), shown as a function of neutrino energy. The solid red line indicates the median performance, whilst the dotted red lines show the region enclosing the central $68 \%$ of events.

The expected sensitivity of the IceCube Upgrade to $v_{\tau}$ appearance is shown in the left panel of Figure 4 , where a precision of $10 \%$ on the $v_{\tau}$ normalisation is achieved after only a single year of data taking, more than $3 \times$ more precise than the current DeepCore result. In addition, the IceCube Upgrade will also be able to measure $v_{\mu}$ disappearance with a precision comparable to current longbaseline accelerator neutrino experiments, at an order of magnitude higher energy. The IceCube Upgrade $v_{\mu}$ disappearance sensitivity after 3 years is shown in the right panel of Figure 4. 

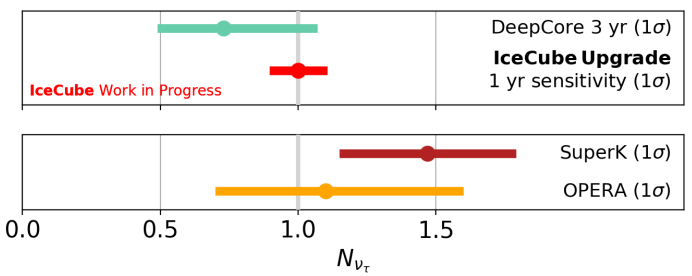

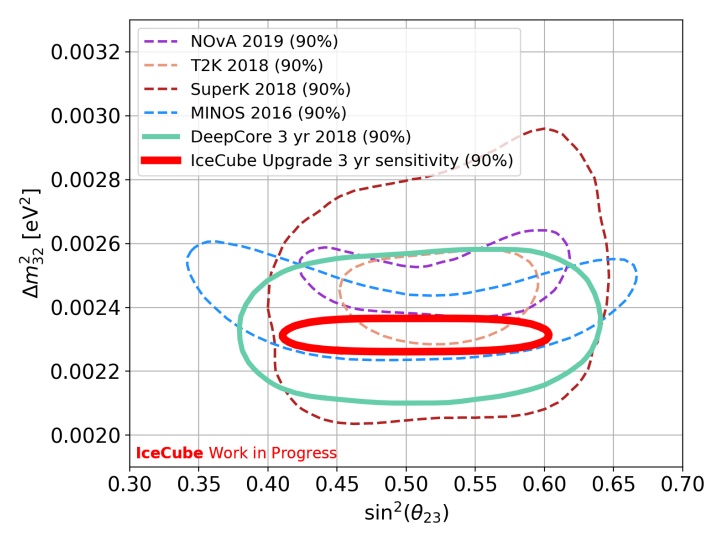

Figure 4: Sensitivity of the IceCube Upgrade to $v_{\tau}$ appearance (left) $v_{\mu}$ disappearance (right), assuming normal neutrino mass ordering and $v$ Fit 4.0 [10] oscillation parameters, with the exception that the atmospheric mixing angle and mass splitting measured in the 2018 DeepCore $v_{\mu}$ disappearance measurement [11] are assumed in the right panel. References to external results can be found in [12].

\section{Conclusions}

Measuring the unitarity of the PMNS neutrino mixing matrix is a powerful test of the $3 v$ paradigm, and measurements of $v_{\tau}$ oscillations are essential to achieve this goal. The DeepCore sub-array of the IceCube neutrino observatory has produced the world's most precise measurement of $v_{\tau}$ appearance in 2019 using three years of data, and the under-construction IceCube Upgrade will provide better than $10 \%$ precision on the $v_{\tau}$ normalisation during the next decade.

The IceCube Upgrade will also feature a broad particle physics program, providing worldleading sensitivity to a range of BSM physics, and pave the way for IceCube Gen2, an expansion of the entire IceCube array initiating a new era in astroparticle physics.

\section{References}

[1] B. Pontecorvo, Sov. Phys. JETP 6, 429 (1957). [Zh. Eksp. Teor. Fiz.33,549(1957)].

[2] Z. Maki, M. Nakagawa, S. Sakata, Progress of Theoretical Physics 28, 870 (1962).

[3] S. Parke, M. Ross-Lonergan, Physical Review D 93 (2016).

[4] N. Agafonova, et al., Phys. Rev. Lett. 120, 211801 (2018).

[5] Z. Li, et al., Physical Review D 98 (2018).

[6] M. Aartsen, et al., Physical Review D 99 (2019).

[7] S. Kretzer, M. H. Reno, Phys. Rev. D 66, 113007 (2002).

[8] M. G. Aartsen, et al., JINST 12, P03012 (2017).

[9] R. Abbasi, et al., Astroparticle Physics 35, 615-624 (2012).

[10] I. Esteban, M. C. Gonzalez-Garcia, A. Hernandez-Cabezudo, M. Maltoni, T. Schwetz, Journal of High Energy Physics 2019, 106 (2019).

[11] M. Aartsen, et al., Physical Review Letters 120 (2018).

[12] A. Ishihara, et al., PoS (ICRC2019) 928 (2019). 